\title{
Efficacy and safety of intraperitoneally administered resveratrol against rat orthotopic ovarian cancers
}

This article was published in the following Dove Press journal: Cancer Management and Research

\author{
Li-Xia Zhong' \\ Mo-Li Wu ${ }^{2}$ \\ Hong $\mathrm{Li}^{2}$ \\ Jia $\mathrm{Liu}^{2}$ \\ Li-Zhu Lin' \\ 'Department of Oncology, The First \\ Affiliated Hospital of Guangzhou \\ University of Chinese Medicine, \\ Guangzhou 5 10407, Guangdong, People's \\ Republic of China; ${ }^{2}$ Liaoning Laboratory \\ of Cancer Genetics and Epigenetics, \\ Department of Cell Biology, College of \\ Basic Medical Sciences, Dalian Medical \\ University, Dalian I I6044, People's \\ Republic of China
}

Background: Resveratrol (Res) inhibits ovarian cancer (OC) cell growth but its in vivo antiOC effects are unclear due to the low bioavailability of systemically administered Res. Intraperitoneal administration may overcome this therapeutic dilemma because it makes Res directly affect the abdominal tumors. Ethanol and DMSO are common Res solvents, while their reliability and safety for long-term in vivo treatment remain unknown.

Methods: A rat orthotopic OC model was established using the rat NUTU-19 OC cell line. Res dissolved in $10 \%$ ethanol or $0.2 \%$ DMSO was injected intraperitoneally $(20 \mathrm{mg} / \mathrm{kg} / \mathrm{day})$ into tumor-free and tumor-bearing rats for 2 weeks. The tumors were collected for gross, morphological and molecular examinations, and blood and ascitic samples were obtained for a CA125 ELISA. Res concentration in ovarian tissues was determined by high performance liquid chromatography (HPLC).

Results: The average tumor weight $(0.187 \pm 0.065 \mathrm{~g})$ of the Res-in-DMSO group was lower than that of untreated $(0.426 \pm 0.091 \mathrm{~g} ; P<0.01)$ and Res-in-ethanol $(0.238 \pm 0.073 \mathrm{~g} ; P<0.05)$ group. The average bloody ascitic volumes collected from untreated, Res-in-ethanol, and Resin-DMSO groups were $5.65 \pm 0.27,2.75 \pm 0.14$, and $2.09 \pm 0.11 \mathrm{ml}$, respectively. Abundant TUNEL-positive cells, ARHI and PIAS3 upregulation, CA125 reduction, and decreased STAT3 nuclear translocation were found in the Res-in-ethanol and, especially, the Res-inDMSO group. Widespread plaques of Res deposits were found on the abdominal serosa of the Res-in-ethanol group, but not in the Res-in-DMSO group. HPLC revealed a higher Res concentration in Res-in-DMSO-treated tumor tissues than in those treated by Res-in-ethanol $(P<0.01)$. Fertility was maintained after long-term Res treatment.

Conclusion: Intraperitoneal administration of Res effectively inhibited rat orthotopic ovarian cancer growth without affecting normal tissues. The Res-in-DMSO group had the highest drug bioavailability and therefore stronger tumor-suppressive effects on ovarian cancer tissues.

Keywords: resveratrol, intraperitoneal administration, rat orthotopic ovarian cancer, apoptosis

\section{Introduction}

Department of Oncology, The First

Affiliated Hospital of Guangzhou

University of Chinese Medicine,

Guangzhou 510407, Guangdong, People's

Republic of China

Email lizhulingz@|26.com

Jia Liu

Department of Cell Biology, College of

Basic Medical Sciences, Dalian Medical

University, Dalian I I6044, People's

Republic of China

Email jialiudl@dmu.edu.cn
Ovarian cancer (OC) is one of the most common malignancies in females ${ }^{1,2}$ with a very poor prognosis because of its subtle symptoms at the early stages of carcinogenesis, ${ }^{3}$ metastasis through blood dissemination, ${ }^{4}$ and peritoneal implantation. ${ }^{5}$ Because of the difficulty to remove advanced ovarian cancers radically, adjuvant chemotherapy has been commonly employed. ${ }^{6}$ Intraperitoneal chemotherapy permits the anticancer drug to directly affect abdominal malignancies and is therefore used in the management of colorectal, liver, gastric and ovarian cancers. ${ }^{7}$ 
Nevertheless, this therapeutic approach also leads to toxic effects. ${ }^{7,8}$ For example, paclitaxel and cisplatin are usually administered intraperitoneally to treat ovarian cancers, but they cause severe side effects including thrombocytopenia and neutrophilia. ${ }^{9,10}$ It would therefore be of clinical significance to explore safer anti-OC agent(s) for intraperitoneal chemotherapy.

A body of evidence has demonstrated that resveratrol exerts the inhibitory effects on in vitro growth of various cancers. ${ }^{11}$ Moreover, the anticancer dose of resveratrol has little adverse effects on normal cells and tissues that possess an efficient metabolic biotransformation system for resveratrol. $^{12,13}$ However, this well-operated resveratrol metabolic system leads to extremely low bioavailability if resveratrol is administered systemically. ${ }^{13,14}$ Therefore, data concerning in vivo anticancer effects of resveratrol are quite limited. ${ }^{11,15}$ It is necessary to overcome this therapeutic bottleneck by organ-targeting drug delivery. In our previous studies, resveratrol was delivered by bladder instillation and lumbar puncture respectively to treated mouse orthotopic bladder cancers ${ }^{12}$ and glioblastomas, ${ }^{16,17}$ which achieved promising results. In view of the extensive application of intraperitoneal chemotherapy in the clinic, the possibilities of intraperitoneal injection to improve the bioavailability of resveratrol in rat ovaries and the therapeutic outcomes of ovarian tumors were elucidated in the current study.

Resveratrol is usually dissolved in $10 \%$ ethanol and administered to the rat abdominal cavity to alleviate doxorubicin-caused hemogram toxicity ${ }^{18}$ and prevent retinal detachment via its neuroprotective effect on light-sensitive cells. ${ }^{19}$ On the other hand, resveratrol dissolved in $0.2 \%$ DMSO is adopted for in vitro studies, ${ }^{14,20}$ but is rarely used in vivo because of the lack of knowledge about the effects of DMSO on normal tissues and organs. Because resveratrol in $0.2 \% \mathrm{DMSO}$ is harmless to cultured normal cells, ${ }^{12,16}$ the absorption, distribution, and safety of intraperitoneally administered resveratrol in $10 \%$ ethanol and $0.2 \%$ DMSO were analyzed and compared using a rat orthotopic ovarian cancer model.

\section{Materials and methods}

\section{Ethic statement}

Prior to the animal experiments, the research protocols had been reviewed and approved by Animal Care and Use Committee of Guangzhou University of Chinese Medicine to guarantee that all studies involving experimental animals were performed in full compliance with National Institutes of Health Guidelines for the Care and Use of Laboratory Animals. 25 female Sprague-Dawley (SD) rats (289 g $\pm 17 \mathrm{~g})$ used in this study were provided by University Experimental Animal Centre and raised under specific pathogen free conditions.

\section{Rat orthotopic ovarian cancer model and intravesical treatment}

NUTU-19 rat poorly differentiated ovarian cancer cell line is derived from the spontaneously transformed Fischer 344 rat ovarian surface epithelial cells. ${ }^{21}$ This cell line was purchased from Shanghai Meixuan Biol Sci Technol Ltd, China and employed for establishing rat orthotopic ovarian cancer model. 21 rats were anesthetized intraperitoneally with $3.5 \%$ chloral hydrate $(0.1 \mathrm{ml} / 10 \mathrm{mg} /$ body weight $)$. The state of anaesthesia was evaluated by loss of righting reflex (LORR), and time to induce LORR was usually between 5-10 mins. In the germ-free isolator, the rat abdominal cavity was opened to expose the ovarian glands; $10 \mu \mathrm{l}\left(5 \times 10^{6}\right)$ NUTU-19 cell suspension ${ }^{21}$ was injected into the sub-epithelial layer of the left gland, and the right side one was cited as normal control. The appearance of "semitransparent bubbles" indicated successful inoculation. Three days later, the rats were randomly divided into three groups (7 rats/group) as Group-1, without treatment; Group-2, treated by $20 \mathrm{mg} / \mathrm{kg}$ Res-in-ethanol; Group-3, treated by $20 \mathrm{mg} / \mathrm{kg}$ Res-in-DMSO. The four tumor-free rats received $20 \mathrm{mg} / \mathrm{kg}$ Res-in-DMSO treatment were used for safety evaluation. The treatments were daily conducted for two weeks.

\section{Sample collection and treatments}

One hour after the final administration, tumor-bearing animals were painlessly sacrificed by an authorized expert at the DMU Animal Center through cervical dislocation. Ascites, whenever present, was extracted from the bottom of pelvic cavity and their volume and cancer cell concentration were measured. Both sides of the ovarian glands were harvested and weighed. Portions of the tumor-containing tissues were snap frozen in liquid nitrogen for frozen sectioning and protein preparation, and the remaining tissues were fixed in $10 \%$ formalin, embedded in paraffin, and sectioned for morphological and immunohistochemical examinations using standard methods. ${ }^{22}$ After the treatment, four resveratrol -treated tumor-free female rats were bred normally with healthy male rats to evaluate their fertility. 


\section{Sample preparation for HPLC analysis}

Samples for HPLC analysis were prepared from frozen tumor specimens and pooled ovarian tissues by a method described elsewhere. ${ }^{13}$ Briefly, a frozen tissue sample was sectioned into $7 \mathrm{~mm}$ slices using a freezing microtome, collected in a centrifuge tube, and extracted with $416 \mu \mathrm{l}$ methanol and $84 \mu \mathrm{l}$ internal standard [IS, 1, 8-dihydroxyanthraquinone, $200 \mu \mathrm{g} / \mathrm{ml}$; (Sigma-Aldrich)]. The tissue homogenates were vortexed for $5 \mathrm{~min}$ and centrifuged at $12,000 \mathrm{rpm}$ for $10 \mathrm{~min}$ at $4{ }^{\circ} \mathrm{C}$. The supernatant was then transferred to a clean tube. The residue was extracted twice with $1 \mathrm{ml}$ methanol by $5 \mathrm{~min}$ of vigorous agitation, followed by centrifugation. The combined organic solvent of the supernatants was evaporated to a final volume of $400 \mu \mathrm{l}$ and subsequently placed in a sealed amber vial for HPLC analysis. ${ }^{23}$ To improve the sensitivity and precision of quantification, 1, 8-dihydroxyanthraquinone was used as the internal standard and trans-resveratrol (SigmaAldrich) as the standard to construct a standard curve using previously described methods. ${ }^{13}$

\section{Histological and immunohistochemical staining}

Paraffin-embedded xenografts were sliced into $7 \mu \mathrm{m}$ sections and subjected to HE morphological staining and immunohistochemical staining by the methods described elsewhere. ${ }^{12}$ Because ARHI has been known as OC suppressor ${ }^{24}$ and STAT3 signaling as the main resveratrol molecular target, ${ }^{25}$ their statuses in the orthotopic tumor tissues with and without resveratrol treatment were analyzed. The antibodies used were mouse anti-ARHI (1:100; Santa Cruz, CA, USA), PIAS3 (1:120; Santa Cruz, CA, USA) and phosphorylated STAT3/p-STAT3 (1:300; ProteinTech, Chicago, IL, USA), respectively. Binding of the primary antibody was detected by a peroxidase reaction using 3,3-diaminobenzidine tetrahydrochloride (DAB) as the substrate (Vector Laboratories, Burlingame, CA, USA). According to the labeling intensity, the staining results were evaluated by two independent researchers and scored as negative (-) if no immunolabeling was observed in target cells, weakly positive $(+)$ if the labeling was faint, moderately positive $(++)$, and strongly positive $(+++)$ when the labeling was stronger or distinctly stronger than $(++)$. Tissue sections without primary antibody incubation were used as background controls for IHC staining and $\beta$-actin was used as a quantitative control for Western blotting.

\section{Tunel-based identification of apoptotic cells}

Cells apoptosis in the tumor tissues was tested by terminal deoxynucleotide transferase(TdT)-mediated dUTP-biotin nick-end labeling (TUNEL) and the apoptotic cells were visualized by DAB colorization and immunofluorescent labeling according to manufacturers' instructions (Roche Diagnostics GmbH, Mannheim, Germany; Promega, Madison, WI, USA). ${ }^{17}$ The immunofluorescent intensities of tumor tissues of the three experimental groups were quantified using ImageJx2 Launcher (Broken Symmetry Software Inc. USA).

\section{Protein extraction and Western blotting}

The frozen tissue samples were sectioned to $7 \mu \mathrm{m}$ slices for protein preparation by the method described elsewhere. ${ }^{22}$ The sample proteins $(50 \mu \mathrm{g} /$ well $)$ were separated in $10 \%$ sodium dodecylsulfate-polyacrylamide gel electrophoresis and transferred to polyvinylidene difluoride membrane (Amersham, Buckinghamshire, UK). The membrane was blocked with 5\% skimmed milk in TBS-T (10 mM Tris- $\mathrm{HCl}, \mathrm{pH} 8.0,150 \mathrm{mM} \mathrm{NaCl}$ and $0.5 \%$ Tween 20) at $4^{\circ} \mathrm{C}$, rinsed 10 mins for three times with TBS-T, followed by $3 \mathrm{~h}$ incubation at room temperature with the first antibody and then $1 \mathrm{~h}$ incubation with HRP-conjugated anti-mouse or anti-rabbit IgG (Zymed Lab, Inc, USA). The bound antibody was detected using the enhanced chemiluminescence system (Roche $\mathrm{GmbH}$, Mannheim, Germany). After removing the labeling signal by incubation with stripping buffer, the membrane was reincubated with other antibodies one by one until all of the parameters (ARHI, PIAS3 and p-STAT3) were examined with the same antibodies used in immunohistochemical staining. $\beta$-actin was cited as the quantitative control.

\section{ELISA analysis of ascetic and serum CAI 25}

Ascetic and serum CA125 levels in individual samples were determined at the end of treatments using an ELISA kit (Lengton Bioscience Co., Ltd., Shanghai, China). Briefly, ascetic samples were incubated in a mouse anti-CA125 antibody-coated 96-well microplate and then developed with a horseradish peroxidase-conjugated secondary antibody. After adding the substrate and then the stop solution, the plates were read on a microplate reader (Thermo Fisher Scientific, Waltham, MA, USA) at a wavelength of $450 \mathrm{~nm}$. The results were calculated based 
on the absorbance of cytokine-antibody complexes. CA125 concentrations were calculated using a standard curve with a $0.1 \mathrm{mg} / \mathrm{mL}$ detection limit.

\section{Statistical analysis}

The experimental data were analyzed by SPSS13.0 statistical software and expressed by mean \pm standard deviation $(\bar{x} \pm \mathrm{s})$. The differences in continuous variables were assessed by Student's $t$-test or one-way ANOVA. Statistical significance was defined as $P<0.05$.

\section{Results}

\section{Improved general states of resveratrol- treated tumor-bearing rats}

The therapeutic outcomes of the two resveratrol groups (Res-in-ethanol and Res-in-DMSO) administered via the intraperitoneal route were elucidated by average body weights, orthotopic tumor weights, ascetic volumes, and ascetic cancer cell numbers. Although no animal died during the experiments, significant loss of average body weight (205.3 $\pm 14 \mathrm{~g} / \mathrm{rat})$ was found in the control group (tumor-bearing animals without treatment) in comparison with normal rats $(289.2 \pm 17.4 \mathrm{~g} / \mathrm{rat} ; P<0.01)$ and
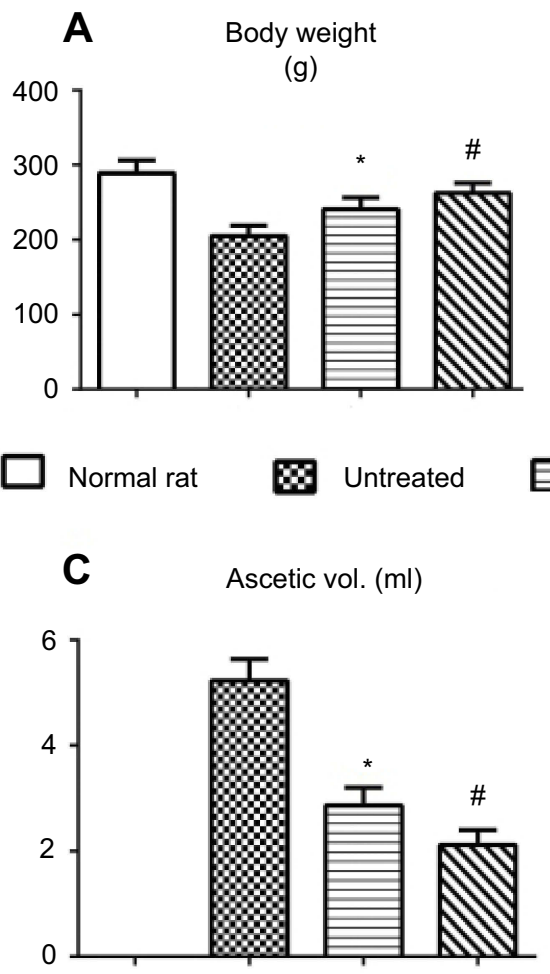
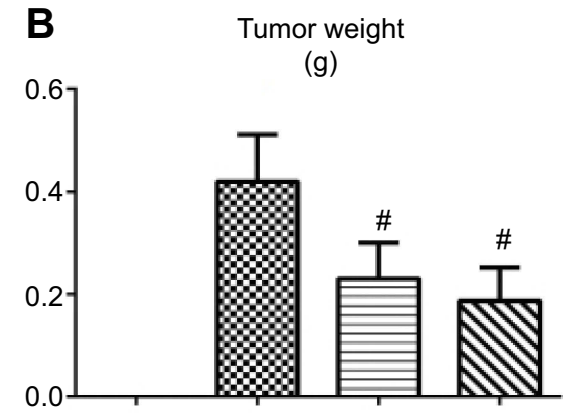

Res-in-ethanol $(241.2 \pm 15.2 \mathrm{~g} / \mathrm{rat} ; P<0.05)$ and Res-inDMSO $(262.9 \pm 13.2 \mathrm{~g} / \mathrm{rat} ; P<0.05)$ rats, respectively (Figure 1A). The average tumor weight was 0.426 $\pm 0.091 \mathrm{~g}$ in the control group, $0.238 \pm 0.073 \mathrm{~g}$ in the Res-in-ethanol group, and $0.187 \pm 0.065 \mathrm{~g}$ in the Res-inDMSO group (Figure 1B). The average volume and cancer cell numbers of abdominal ascites were 5.65 $\pm 0.27 \mathrm{ml}$ and $2.8 \times 10^{4} / \mathrm{ml}$ in the control group, 2.75 $\pm 0.14 \mathrm{ml}$ and $1.4 \times 10^{4} / \mathrm{ml}$ in the Res-in-ethanol group, and $2.09 \pm 0.11 \mathrm{ml}$ and $0.95 \times 10^{4} / \mathrm{ml}$ in the Res-in-DMSO group, respectively (Figure $1 \mathrm{C}$ and $\mathrm{D}$ ).

\section{Resveratrol availability in tumor tissues}

HPLC analysis revealed that the amounts of resveratrol in orthotopic ovarian cancer tissue and its tumor-free ovarian counterpart of individual rats were determined one hour after final trans-resveratrol i.p. administration. As shown in Figure 2A, only one peak corresponding to trans-resveratrol could be detected in the tumor tissue extracts. The average resveratrol concentration was $1.237 \pm 0.203 \mathrm{nmol} / \mathrm{g}$ in Res-in-ethanol treated tumor tissues and $2.154 \pm 0.287 \mathrm{nmol} / \mathrm{g}$ in Res-in-DMSO treated ones $(P<0.01$; Figure $2 \mathrm{~B})$.

Res-in-ethanol

$\mathbf{D}$ Res-in-DMSO
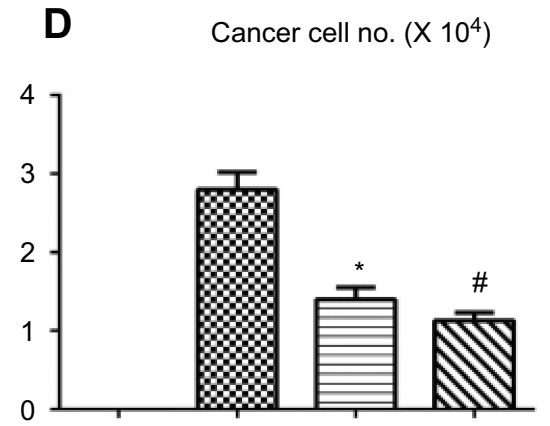

Figure I Resveratrol improves general states of tumor-bearing rats. General states of tumor-bearing rats without treatment (Untreated) and treated with resveratrol in $10 \%$ ethanol (Res-in-Ethanol) or resveratrol in $0.2 \%$ DMSO (Res-in-DMSO) by evaluating average body weights (A), orthotopic tumor weights (B), ascetic volumes (C), and ascetic cancer cell numbers (D). The data obtained was validated by Student's $t$-test. $* P<0.05$ and ${ }^{\# P<0.01}$ in comparison with the untreated group.

Abbreviation: Res, resveratrol. 
A

B

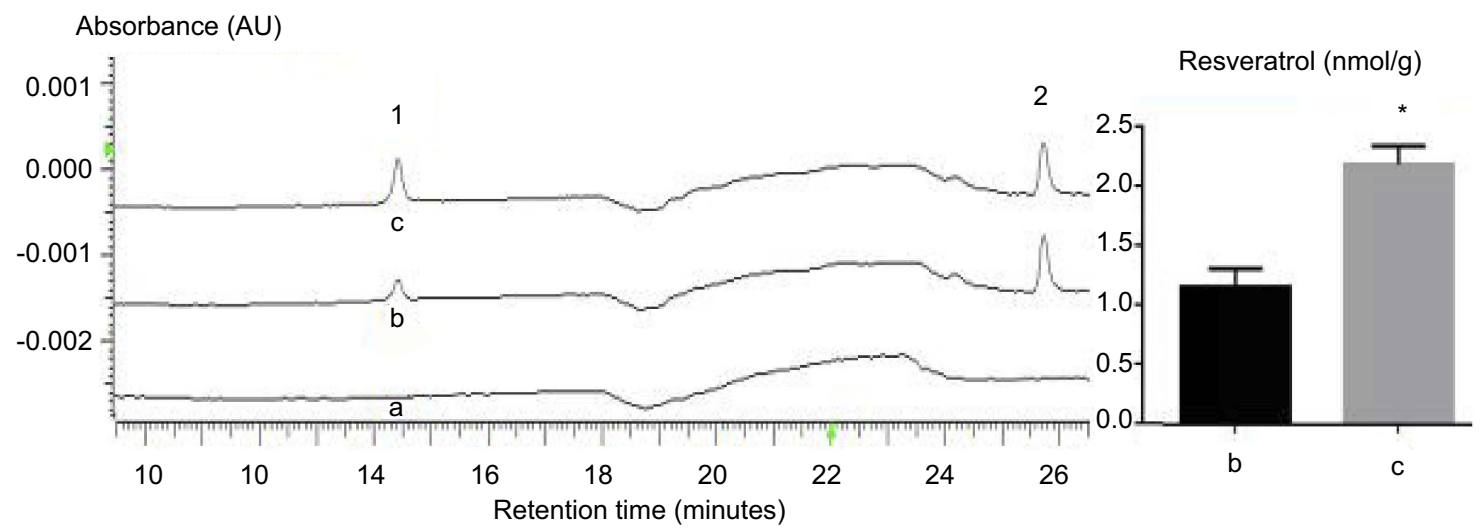

Figure 2 Sufficient resveratrol in orthotopic tumor tissues. (A and B) HPLC/DAD demonstration of retention times and absorbance of i.p. administered Res-in-ethanol and Res-in-DMSO in orthotopic ovarian cancer tissues. Peak-I, trans-resveratrol; Peak-2: I,8-2 hydroxy anthraquinone as internal standard. (a) without treatment as the negative control; (b) Res-in-ethanol; (c) Res-in-DMSO. Student's t-test was used to validate the obtained data. $* P<0.01$ in comparison with $b$.

Abbreviations: AU, absorbance retention time; Res, resveratrol; HPLC, high performance liquid chromatography; DAD, diode array detector.

\section{Resveratrol plaques on abdominal mucosa of Res-in-ethanol rats}

Animals were painlessly sacrificed at $1 \mathrm{hr}$ after the last resveratrol injection. White plaques were widely distributed in the abdominal mucosa of Res-in-ethanol rats, but not in that of Res-in-DMSO rats (Figure 3A). HPLC analysis of the collected white plaques revealed a single peak corresponding to trans-resveratrol (Figure 3B), confirming that the plaques were deposits of precipitated resveratrol.

\section{Resveratrol induced apoptosis in cancer but not noncancerous tissues}

$\mathrm{H} \& \mathrm{E}$ staining revealed distinct morphological changes of the tumor tissues of Res-in-ethanol and, especially, Res-in-DMSO group in terms of abundant distribution of tumor cells with shrinkage and chromatin condensation (Figure 4A). TUNEL apoptosis assay by DAB and immunofluorescent coloration demonstrated the abundant TUNEL-positive cells (Figure 4A and B) and remarkably increased IF pixel values (Figure 4C) in resveratrol-treated tumor tissues particularly that in the Resin-DMSO group. Neither cell death nor morphological alteration was found in both the noncancerous ovarian tissues or the tissues of the heart, liver, spleen, lungs, kidneys and intestine (Figure 5).

\section{Resveratrol suppressed STAT3 transcription and activation}

The effects of resveratrol on STAT3 signaling in the orthotopic tumor tissues of the experimental groups were analyzed by immunohistochemical staining and Western blotting. The results revealed that STAT3 was expressed in the untreated tumor tissues with remarkable nuclear translocation (Figure 6A). In contrast, phosphoralated-STAT3 (p-STAT3) nuclear translocation became infrequent after resveratrol treament (Figure 6A). Down-regulated $(24.5 \%)$ STAT3 expression with the reduced phosphoralated-STAT3 generation $(48.5 \%)$ were evidenced in the tumor tissues of Res-in-ethanol group and became more distinct (83.1\% and $81.6 \%$ ) in that of Res-in-DMSO group (Figure 6B).

\section{ARHI and PIAS3 upregulation in resveratrol-treated tumors}

ARHI and PIAS3 are known as the suppressors of STAT3 signaling. ${ }^{24,25}$ Immunocytochemical staining demonstrated that the levels of these two proteins were very low in the untreated orthotopic tumor tissues, which were distinctly increased by resveratrol dissolved in $10 \%$ ethanol and especially in $0.2 \%$ DMSO (Figure $7 \mathrm{~A}$ ). The results of Western blotting were in accordance with the ICC findings by showing 4.2 fold and 7.6 fold increases of ARHI and PIAS3 levels in the tumor samples of the Res-in-ethanol group and 6.5 fold and 9.1 fold increases of the two proteins in Res-in-DMSO group (Figure 7B).

\section{Resveratrol reduces ascetic and serum CAI 25 levels}

A increased serum CA125 level is an unfavorable prognostic indicator of ovarian cancer patients. $^{26}$ As shown in Figure 8A, the average serum CA125 level of the untreated 
A

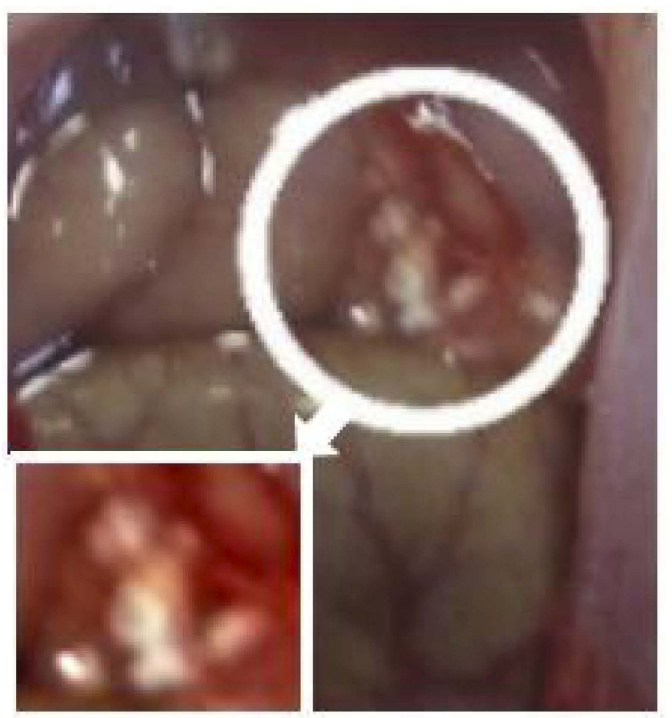

Res-in-DMSO

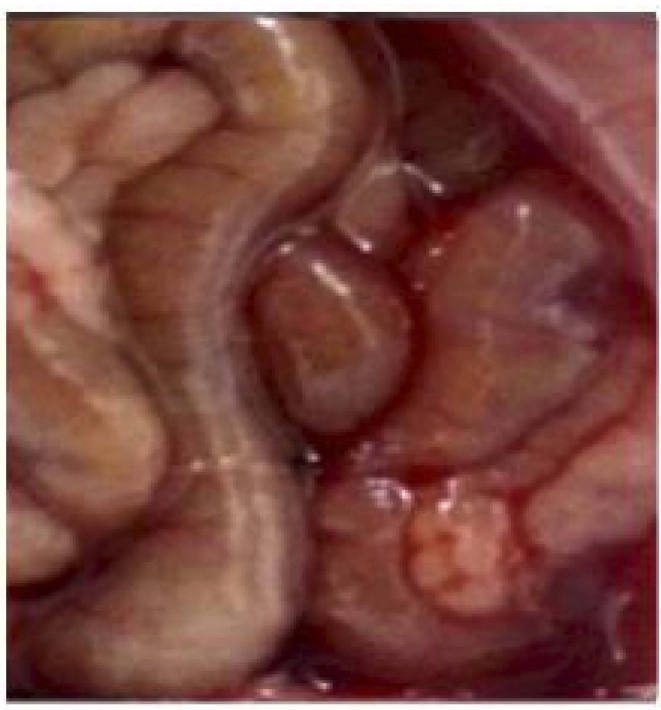

B

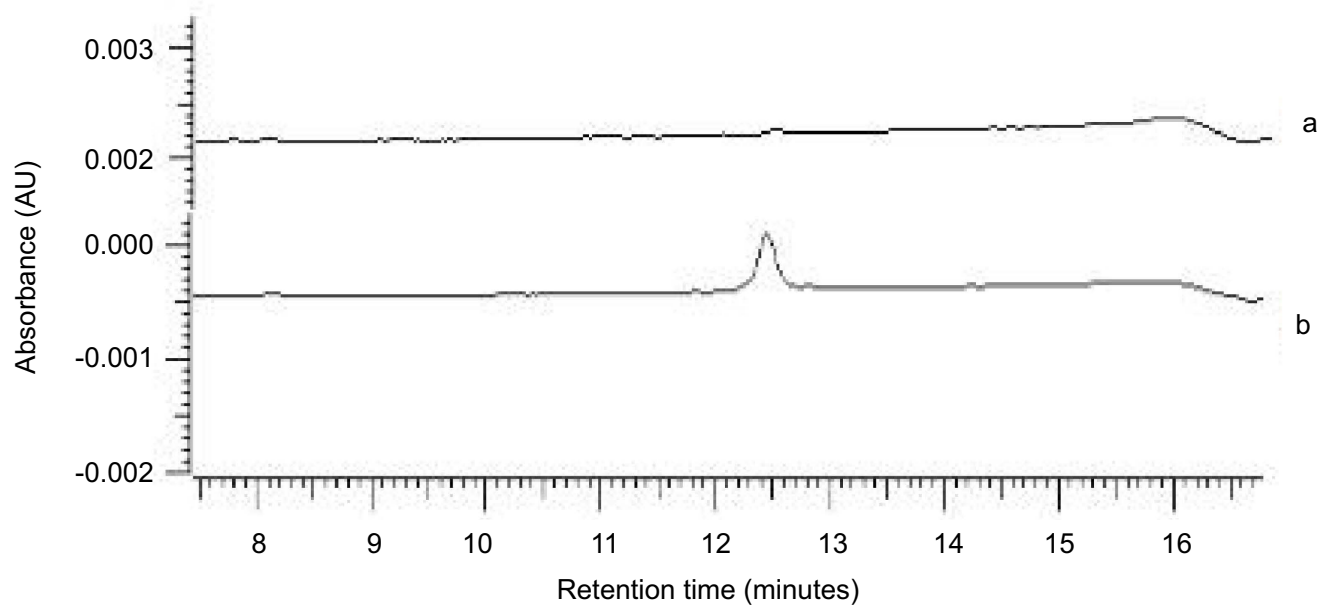

Figure 3 HPLC identification of trans-resveratrol in abdominal white plaques of Res-in-ethanol-treated rats. (A) Arrow indicates widely distributed white plaques on the surface of the peritoneal serosa of Res-in-ethanol treated rats (left), but not in Res-in-DMSO-treated rats (right). Inset, circled region at higher magnification ( $\times 80)$. (B) HPLC analysis of collected white plaques revealed a single peak that corresponded to trans-resveratrol. a, Control; b. Res-in-ethanol.

Abbreviations: $\mathrm{AU}$, absorbance retention time; HPLC, high performance liquid chromatography; Res, resveratrol.

group was $8.606 \pm 0.819 \mathrm{ng} / \mathrm{mL}$, which was significantly decreased to $4.132 \pm 0.311 \mathrm{ng} / \mathrm{mL} \quad(p<0.01)$ and 3.769 $\pm 0.285 \mathrm{ng} / \mathrm{mL}(P<0.01)$ in Res-in-ethanol and Res-inDMSO groups, respectively. The average CA125 concentration was $6.125 \mathrm{ng} / \mathrm{mL} \pm 0.402$ in the ascetic samples of the untreated group in comparison with $5.177 \mathrm{ng} / \mathrm{mL} \pm 0.371$ in the Res-in-ethanol group and $4.245 \mathrm{ng} / \mathrm{mL} \pm 0.287$ in the Res-in-DMSO group $(P<0.05)$. As shown in Figure $8 \mathrm{~B}$, the CA125 content in total ascites of the untreated group was $19.624 \mathrm{ng} \pm 0.743 / 3.12 \mathrm{~mL}$, which was higher $(P<0.05)$ than that of Res-in-ethanol group $(14.237 \mathrm{ng} \pm 0.612 / 2.75 \mathrm{~mL})$ and significantly higher $(P<0.01)$ than that of the Res-in-DMSO group (8.872 $\mathrm{ng} \pm 0.7369 / 2.09 \mathrm{~mL})$.

\section{Maintained fertility after 2 weeks of resveratrol treatment}

Four tumor-free female rats were bred normally with healthy male rats at a 1:1 ratio after the same dose $(20 \mathrm{mg} / \mathrm{kg} /$ day $)$ of intraperitoneal Res-in-DMSO treatment for 2 weeks. After 34-37 days, the four female animals successfully produced 12-14 pups, and all juvenile rats grew normally without any visible abnormalities (data not shown).

\section{Discussion}

Ovarian cancer is the most lethal genital malignancy. The survival rate of ovarian cancer patients has been improved 


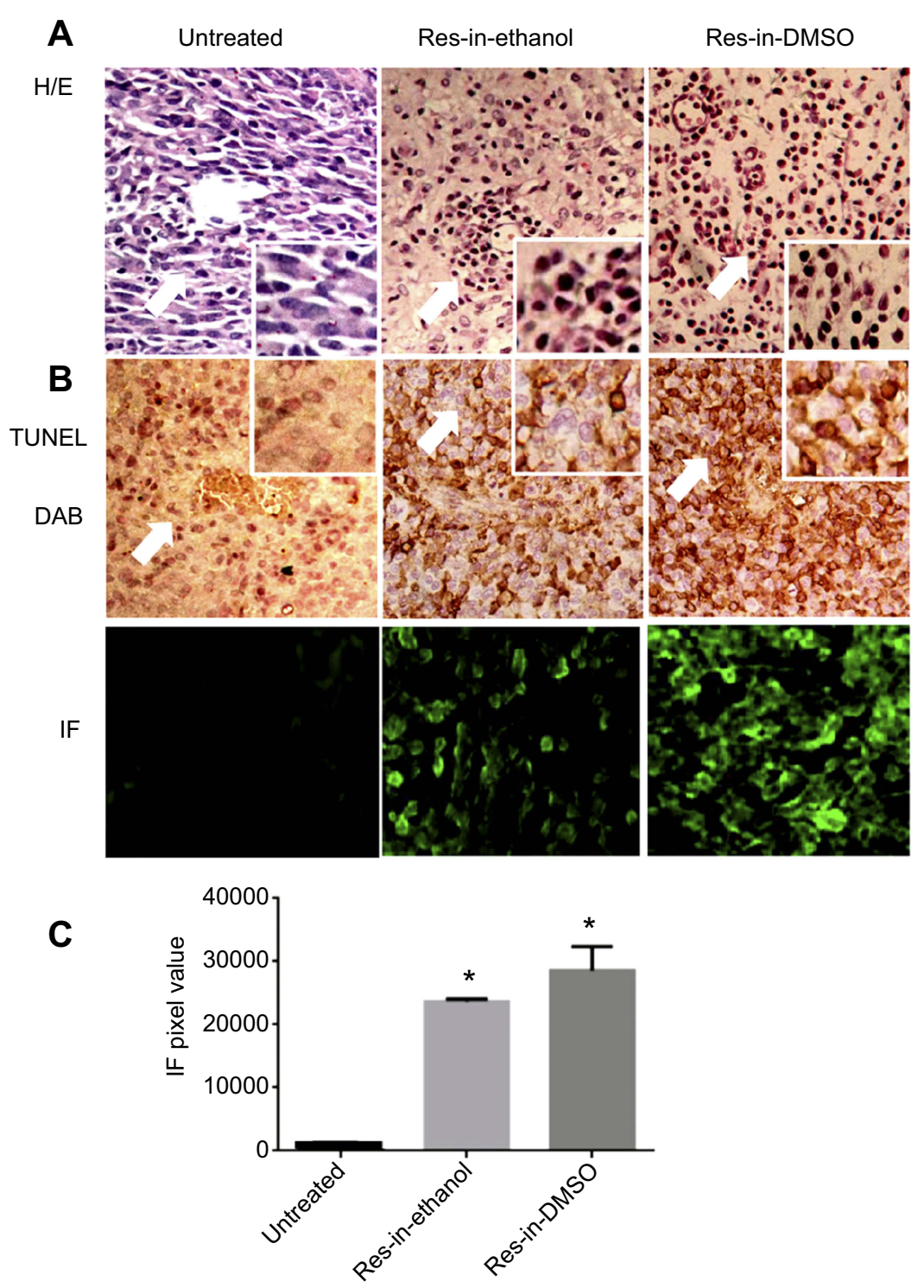

Figure 4 Resveratrol induces apoptosis in ovarian cancer tissues. (A) H\&E morphological staining revealed more aggressive tumor growth of the untreated group. Extensive cell death was found in tumor tissues of both Res-in-ethanol and Res-in-DMSO groups. Arrows indicate regions with higher magnification in insets ( $\times 40$ ). (B) DAB colorization and IF labeling of apoptotic cells in resveratrol-treated orthotopic tumor tissues by a TUNEL apoptosis assay $(\times 20)$. Arrows indicate regions with higher magnification in insets $(\times 40)$. (C) Image $\times 2$-based evaluation of TUNEL immunofluorescent pixel values of untreated tumor tissues and the tumor tissues treated by Res-inethanol and Res-in-DMSO. ANOVA was used to validate these data. $* P<0.01$ in comparison with untreated tumor tissues.

Abbreviations: DAB, 3,3-diaminobenzidine tetrahydrochloride ; IF, immunofluorescence; Res, resveratrol.

somewhat, but it is largely attributed to early detection by advanced diagnostic techniques rather than the revolutionary progress of adjuvant treatments. ${ }^{27}$ Consequently, the prognosis of patients with advanced ovarian cancer remains poor because of the lack of effective postoperative therapies. ${ }^{28}$ It is therefore of great clinical value to explore new drugs for ovarian cancer treatment. Resveratrol effectively inhibits the growth of ovarian cancer cells in vitro without affecting normal tissues and cells, ${ }^{29-31}$ indicating its natural propensity as an ideal anticancer drug. However, this polyphenol compound has not yet been applied to clinical practice, because it can be easily metabolized in vivo and quickly expelled from the body. ${ }^{13}$ Intraperitoneal injection is one of the conventional therapeutic approaches for ovarian cancer, by which the administered drug directly affects the tumor to inhibit its growth, metastasis, and recurrence. ${ }^{32}$ We therefore consider that such administration is suitable for resveratrol in OC treatment. Ethanol and DMSO are frequently used solvents for resveratrol, but there is a lack of comparative analysis of the absorption rates, anticancer efficacy and safety of the two resveratrol preparations in vivo. To assess the efficacy and safety of intraperitoneal 


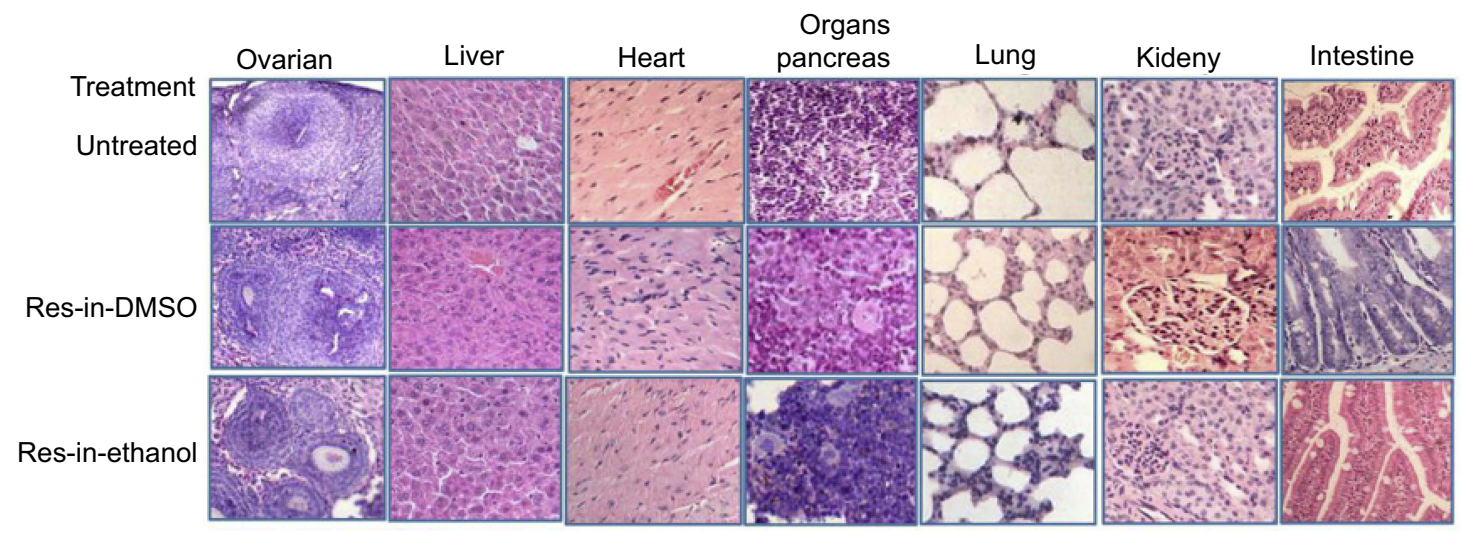

Figure $5 \mathrm{H} \mathrm{\&} \mathrm{E} \mathrm{staining} \mathrm{showed} \mathrm{neither} \mathrm{cell} \mathrm{death} \mathrm{nor} \mathrm{morphological} \mathrm{alteration} \mathrm{in} \mathrm{the} \mathrm{noncancerous} \mathrm{ovarian} \mathrm{tissues} \mathrm{and} \mathrm{the} \mathrm{tissues} \mathrm{of} \mathrm{the} \mathrm{heart,} \mathrm{liver,} \mathrm{spleen,} \mathrm{lungs,} \mathrm{kidneys}$ and intestine of the tumor-bearing rats without drug treatment (Untreated), treated by Res-in-DMSO or Res-in-Ethanol.

Abbreviation: Res, resveratrol.

A

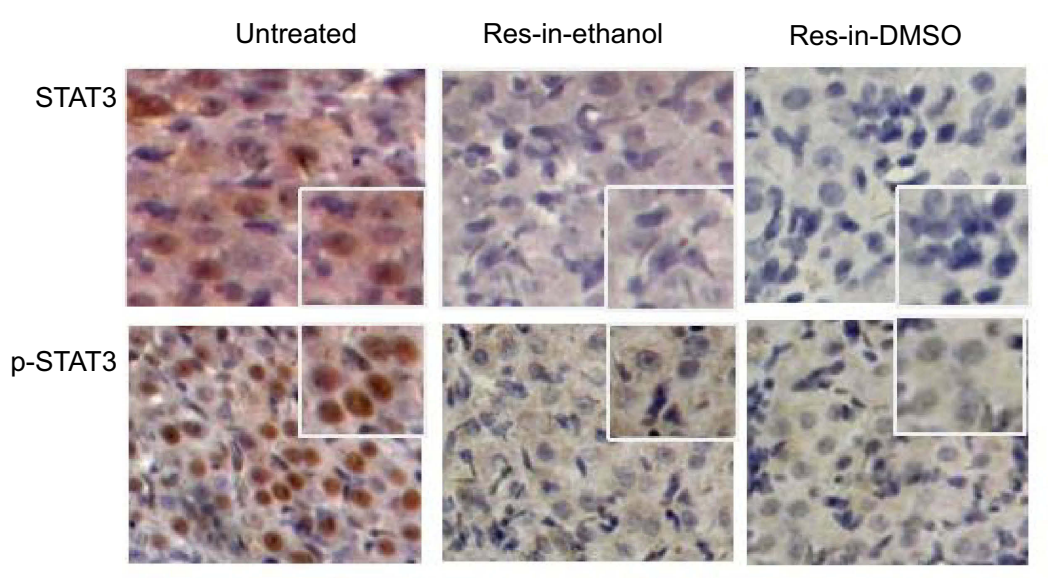

B

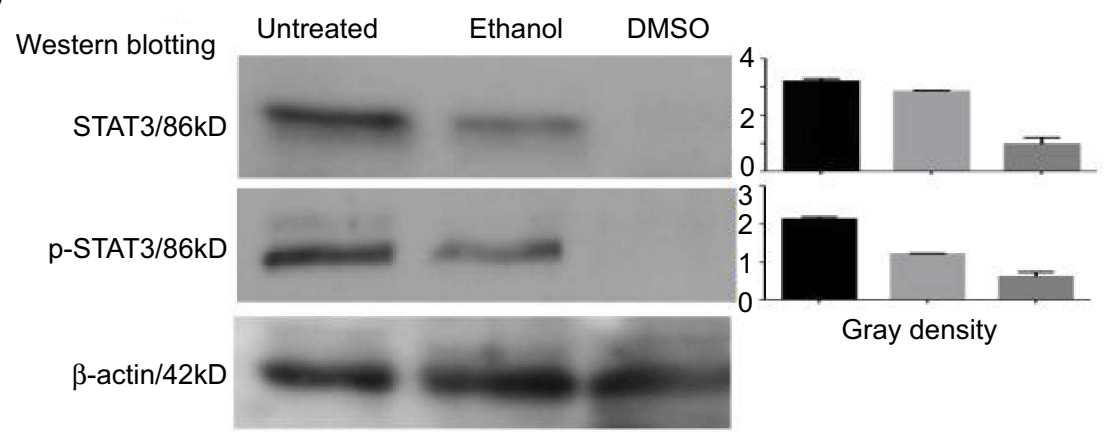

Figure 6 Resveratrol suppresses STAT3 activation in orthotopic ovarian cancer tissues. STAT3 transcription and nuclear translocation in ovarian cancer tissues were elucidated by immunohistochemical staining (A) and Western blotting. (B) Tumor samples were collected from untreated rats (Untreated) and rats treated with resveratrol in $10 \%$ ethanol (Res-in-ethanol) or $0.2 \%$ DMSO (Res-in-DMSO). Insets, $\times 40$ magnification.

Abbreviation: Res, resveratrol.

resveratrol administration for ovarian cancer treatment, a rat orthotopic ovarian cancer model was established using the rat NUTU-19 OC cell line ${ }^{21}$ and applying resveratrolbased experimental treatments by imitating clinical intraperitoneal chemotherapy.
As the first step of our study, the bioavailabilities of i.p. administered resveratrol dissolved in 10\% ethanol (Res-inEthanol) or $0.2 \%$ DMSO (Res-in-DMSO) were elucidated by HPLC analysis. Only trans-resveratrol was detected in the samples. This finding suggests that, unlike the fate of 
A

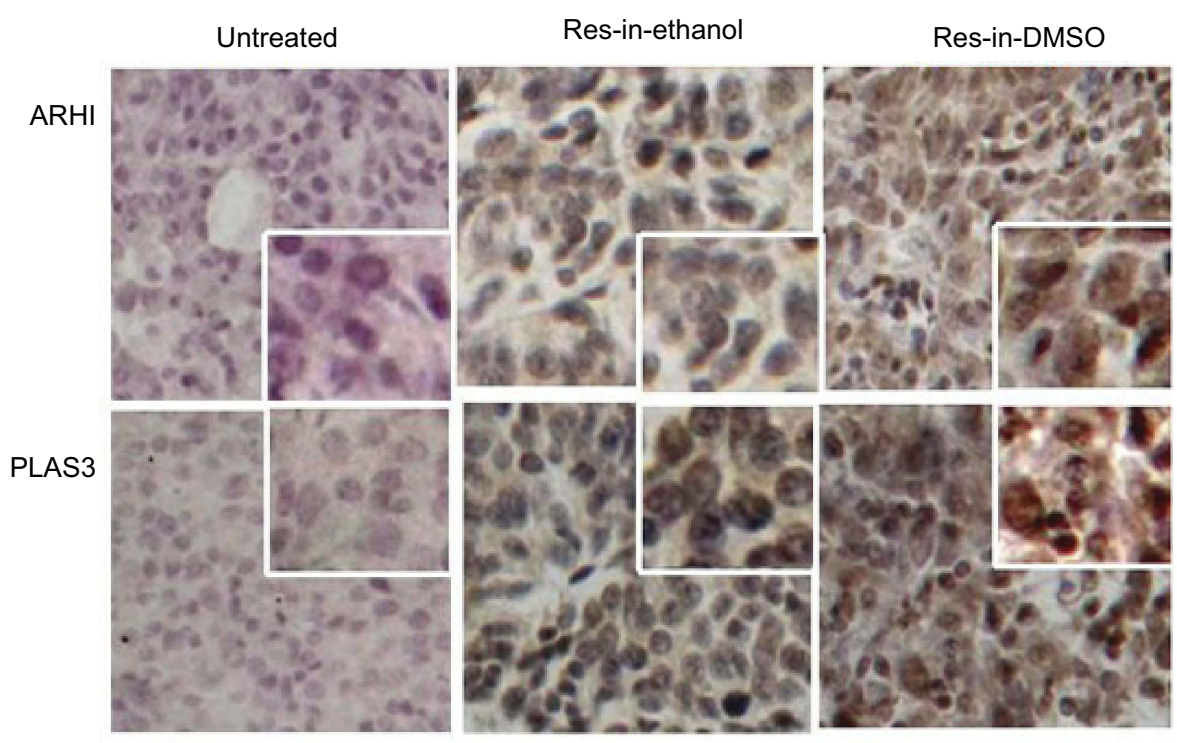

B

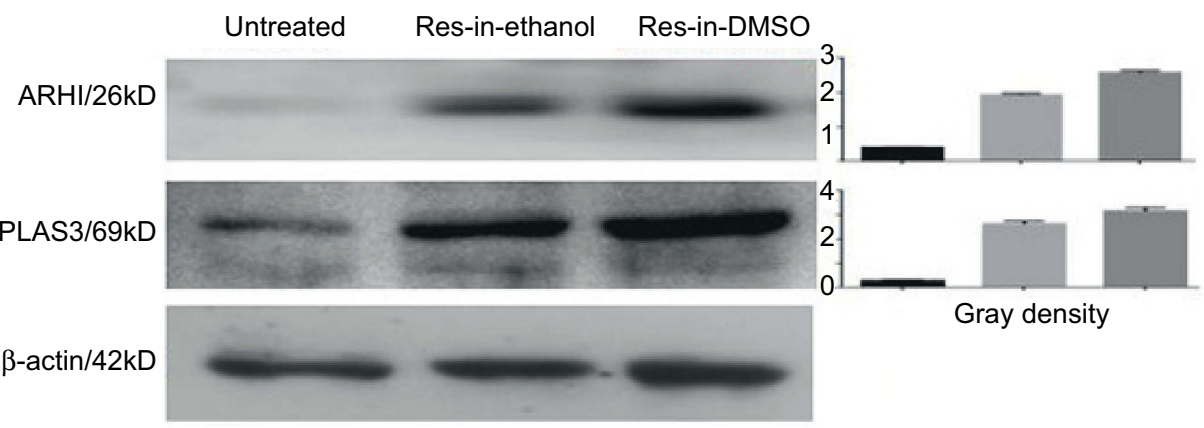

Figure $7 \mathrm{ARHI}$ and PIAS3 upregulation in resveratrol-treated tumors. Immunocytochemical staining (A) and Western blotting (B) were conducted to analyze ARHI and PIAS3 expression in tumor tissues of untreated, resveratrol in 10\% ethanol (Res-in-ethanol), and resveratrol in $0.2 \%$ DMSO (Res-in-DMSO) groups. Insets, $\times 40$ magnification.

Abbreviation: Res, resveratrol.

systemically administered trans-resveratrol, ${ }^{13,33}$ the chemical structure of this compound is well maintained in ovarian tissue when delivered via the i.p. route. Moreover, the average resveratrol concentration in orthotopic tumor tissues of the Res-in-DMSO group was 1.74 times higher than that of the Res-in-ethanol group, indicating a better absorption rate and anticancer effects of the $0.2 \%$ DMSO-dissolved resveratrol solution. The reason for the different bioavailabilities of the two resveratrol solutions can be explained by the widespread white plaques found on the abdominal mucosa of the Res-in-ethanol group, which were identified as trans-resveratrol precipitates. More importantly, no morphological alteration was observed in major organs of rats, and female rats maintained their reproductive ability after long-term Res-in-DMSO treatment. Therefore, the safety and absorptive efficiency of i.p. administration of this reagent were clarified, which may be more suitable for in vivo therapeutic study.
Because 2 weeks of intraperitoneal resveratrol treatment at a daily dose of $20 \mathrm{mg} / \mathrm{kg}$ had few adverse effects on rat health, this therapeutic strategy was applied to treat rat orthotopic ovarian cancers. Untreated tumor-bearing rats showed weight loss and a full abdomen in comparison with their healthy counterparts. There symptoms also occurred in rats of the two treatment groups but the degrees were less significant. Anatomical examination revealed that the average tumor weight, extracted ascites volume, ascitic cancer cell concentration, and CA125 level of untreated tumor-bearing rats were significantly higher than those of the Res-in-Ethanol group and, especially, the Res-in-DMSO group. These results demonstrated that the i.p. administered resveratrol efficiently inhibited the growth and progression of the tumors and therefore improved the general condition of the tumor-bearing rats. The suitability of DMSO-dissolved resveratrol was further confirmed because of the more effective inhibitory effects and higher resveratrol 


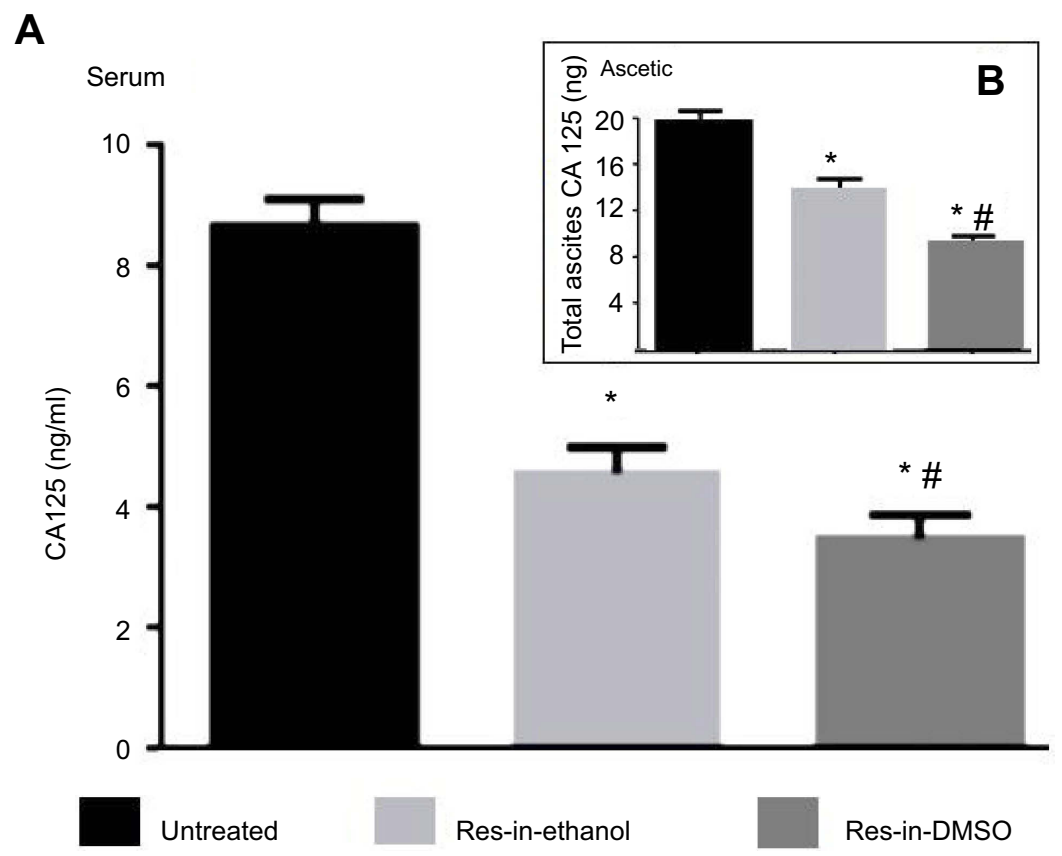

Figure 8 Decreased serum and ascetic CAI 25 levels in resveratrol-treated tumor-bearing rats. A CAI25 ELISA was performed using the serum (A) and ascetic samples (B) of tumor-bearing rats without treatment (Untreated) and treated by intraperitoneal administration of resveratrol dissolved in $10 \%$ ethanol (Res-in-ethanol) or $0.2 \%$ DMSO (Res-in-DMSO). Student's $t$-test was used to validate the obtained data. *, $P<0.05$ in comparison with the untreated group;,$P>0.05$ in comparison with the Res-in-ethanol group.

Abbreviation: Res, resveratrol.

bioavailability in the Res-in-DMSO group. It should be pointed out that i.p. resveratrol chemotherapy by itself would still be insufficient to cure orthotopic tumors. Because chemotherapy is conventionally used postoperatively to prevent ovarian cancer relapse and metastasis, ${ }^{34,35}$ it would be worthwhile to elucidate whether the therapeutic outcome can be further improved by the combination of surgery and i.p. resveratrol administration similarly to what has been shown in a rat orthotopic glioblastoma model. ${ }^{13,16,17}$

A body of evidence has demonstrated that resveratrol exerts multifaceted biological effects on cultured OC cells, including apoptosis induction, ARH1 upregulation, and inactivation of STAT3 signaling [24, 30, 31]. In view of the importance of these events in determining the fate of $\mathrm{OC}$ cells, they were used as key parameters to evaluate the therapeutic efficacy and anti-OC mechanism of i. p. resveratrol administration. In accordance with our previous in vitro findings, ${ }^{30,31}$ the protein level and incidence of nuclear translocation of STAT3 were reduced in Res-in-Ethanol- and, more significantly, in Res-in-DMSO-treated tumor tissues with obvious apoptosis. STAT3 signaling is critical for the growth and survival of OC cells, because selective inhibition of its activation commits OC cells to apoptosis. ${ }^{24}$ In this context, the inactivated STAT3 signaling in resveratrol-treated tumor tissues provides molecular evidence for the effectiveness of i. $p$. administered resveratrol. This notion is further supported by upregulation of ARHI and PIAS3, because ARHI is a suppressor of OC cell proliferation ${ }^{24,36}$ and STAT3 signaling, ${ }^{37}$ and PIAS3 is an inhibitor of STAT3 transcriptional activity by blocking the STAT3 DNA-binding domain. ${ }^{38}$

\section{Conclusions}

In this study, the bioavailability, safety, and tumorsuppressive effect of i. p. administered resveratrol dissolved in $10 \%$ ethanol or $0.2 \%$ DMSO were investigated in a rat orthotopic ovarian cancer model. The results revealed that resveratrol in DMSO had a better absorption rate and long-term safety in rat major organs, and preserved reproductive capacity. Resveratrol in DMSO more efficiently inhibited orthotopic tumor growth in terms of distinct reduction of tumor sizes, ascitic formation, ascitic cancer cell numbers, and CA125 levels. Abundant TUNEL-positive cells, downregulated STAT3 expression, decreased pSTAT3 nuclear translocation and ARHI upregulation were found in resveratrol-treated tumor tissues, providing cellular and molecular evidence for the anti-OC 
reliability of this therapeutic approach, especially when employing a resveratrol in DMSO solution.

\section{Acknowledgments}

This work was supported by National Fund for Postdoctoral fellows (No. 2017M622668) and the grants from National Natural Science Foundation of China (No. 81573780, 81450016, 81272786 and 81071971).

\section{Disclosure}

The authors report no conflicts of interest in this work.

\section{References}

1. Lowe KA, Chia VM, Taylor A, O’Malley C, Kelsh M, Mohamed M. An international assessment of ovarian cancer incidence and mortality. Gynecol Oncol. 2013;130:107-114. doi:10.1016/j.ygyno.2013.03.026

2. Menon U, Ryan A, Kalsi J, et al. Risk algorithm using serial biomarker measurements doubles the number of screen-detected cancers compared with a single-threshold rule in the United Kingdom collaborative trial of ovarian cancer screening. $J$ Clin Oncol. 2015;33:2062-2071. doi:10.1200/JCO.2014.59.4945

3. Menon U, Gentry-Maharaj A, Hallett R, et al. Sensitivity and specificity of multimodal and ultrasound screening for ovarian cancer, and stage distribution of detected cancers: results of the prevalence screen of the UK Collaborative Trial of Ovarian Cancer Screening (UKCTOCS). Lancet Oncol. 2009;10:327-340. doi:10.1016/S1470-2045(09)70026-9

4. Yeung TL, Leung CS, Yip KP, Au Yeung CL, Wong ST, Mok SC. Cellular and molecular processes in ovarian cancer metastasis. A review in the theme: cell and molecular processes in cancer metastasis. Am J Physiol Cell Physiol. 2015;309:C444-C456. doi:10.1152/ajpcell.00213.2015

5. Justyna MP, Paweł U, Andrzej T, Krzysztof K. The peritoneal "soil" for a cancerous "seed": a comprehensive review of the pathogenesis of intraperitoneal cancer metastases. Cell Mol Life Sci. 2018;75:509525. doi:10.1007/s00018-017-2663-1

6. Wright AA, Bohlke K, Armstrong DK, et al. Neoadjuvant chemotherapy for newly diagnosed, advanced ovarian cancer: Society of Gynecologic Oncology and American Society of Clinical Oncology Clinical Practice Guideline. J Clin Oncol. 2016;34:3460-3473. doi:10.1200/JCO.2016.68.6907

7. Cortez AJ, Tudrej P, Kujawa KA, Lisowska KM. Advances in ovarian cancer therapy. Cancer Chemother Pharmacol. 2018;81:17-38 doi:10.1007/s00280-017-3465-8

8. Westrøm S, Bønsdorff TB, Bruland ØS, Larsen RH. Therapeutic effect of $\alpha$-Emitting 224Ra-labeled calcium carbonate microparticles in mice with intraperitoneal ovarian cancer. Transl Oncol. 2018;11:259-267. doi:10.1016/j.tranon.2017.10.004

9. Micha JP, Goldstein BH, Mattison JA, et al. Experience with singleagent paclitaxel consolidation following primary chemotherapy with carboplatin, paclitaxel, and gemcitabine in advanced ovarian cancer. Gynecol Oncol. 2005;96:132-135. doi:10.1016/j.ygyno.2004.10.001

10. Havrilesky LJ, Secord AA, Darcy KM, Armstrong DK, Kulasingam S. Gynecologic Oncology Group: cost effectiveness of intraperitoneal compared with intravenous chemotherapy for women with optimally resected stage III ovarian cancer: a Gynecologic Oncology Group Study. J Clin Oncol. 2008;26:4144 4150. doi:10.1200/JCO.2007.15.2777

11. Singh CK, Ndiaye MA, Ahmad N. Resveratrol and cancer: challenges for clinical translation. Biochim Biophys Acta. 2015;1852:1178-1185. doi:10.1016/j.bbadis.2015.08.002
12. Wu ML, Li H, Yu LJ, et al. Short-term resveratrol exposure causes in vitro and in vivo growth inhibition and apoptosis of bladder cancer cells. PLoS One. 2014;9:e89806. doi:10.1371/journal.pone.0089806

13. Shu XH, Wang LL, Li H, et al. Diffusion efficiency and bioavailability of resveratrol administered to rat brain by different routes: therapeutic implications. Neurotherapeutics. 2015;12:491-501. doi:10.1007/s13311-014-0334-6

14. Shu XH, Li H, Sun Z, et al. Identification of metabolic pattern and bioactive form of resveratrol in human medulloblastoma cells. Biochem Pharmacol. 2010;79:1516-1525. doi:10.1016/j.bcp.2010.01.022

15. Carter LG, D’Orazio JA, Pearson KJ. Resveratrol and cancer: focus on in vivo evidence. Endocr Relat Cancer. 2014;21:R209-R225. doi:10.1530/ERC-13-0171

16. Song X, Shu XH, Sha L, et al. Lumbar puncture-administered resveratrol inhibits STAT3 activation, enhancing autophagy and apoptosis in orthotopic rat glioblastomas. Oncotarget. 2016;7(46):7579075799. doi:10.18632/oncotarget. 12414

17. Song X, Shu XH, Wu ML, et al. Postoperative resveratrol administration improves prognosis of rat orthotopic glioblastomas. $B M C$ Cancer. 2018;18:871. doi:10.1186/s12885-018-4242-8

18. Shoukry HS, Ammar HI, Rashed LA, et al. Prophylactic supplementation of resveratrol is more effective than its therapeutic use against doxorubicin induced cardiotoxicity. PLoS One. 2017;12:181535. doi:10.1371/journal.pone.0181535

19. Huang W, Li G, Qiu J, Gonzalez P, Challa P. Protective effects of resveratrol in experimental retinal detachment. PLoS One. 2013;8: e75735. doi:10.1371/journal.pone. 0075735

20. Buhrmann C, Shayan P, Goel A, Shakibaei M. Resveratrol regulates colorectal cancer cell invasion by modulation of focal adhesion molecules. Nutrients. 2017;9:1073. doi:10.3390/nu9101073

21. Rose GS, Tocco LM, Granger GA, et al. Development and characterization of a clinically useful animal model of epithelial ovarian cancer in the fischer 344 rat. Am J Obstet Gynecol. 1996;175(3 Pt 1):593-599.

22. Li C, Li H, Zhang P, et al. SHP2, SOCS3 and PIAS3 expression patterns in medulloblastomas: relevance to STAT3 activation and resveratrol-suppressed STAT3 signaling. Nutrients. 2017;15:847-852.

23. Sun Z, Li H, Shu XH, et al. Distinct sulfonation activities in resveratrol-sensitive and resveratrol-insensitive human glioblastoma cells. Febs J. 2012;279:2381-2392. doi:10.1111/j.1742-4658.2012.08617.x

24. Zhong LX, Nie JH, Liu J, Lin LZ. Correlation of ARHI upregulation with growth suppression and STAT3 inactivation in resveratrol-treated ovarian cancer cells. Cancer Biomarkers. 2018;21:787-795. doi:10.3233/CBM-170483

25. Yu LJ, Wu ML, Li H, et al. Inhibition of STAT3 expression and signaling in resveratrol-differentiated medulloblastoma cells. Neoplasia. 2008;10 (7):736-744.

26. Esselen KM, Cronin AM, Bixel K, et al. Use of CA-125 tests and CT scans for surveillance in ovarian cancer. JAMA Oncol. 2016;2:14271433. doi:10.1001/jamaoncol.2015.5778

27. Cornelison R, Llaneza DC, Landen CN. Emerging therapeutics to overcome chemoresistance in epithelial ovarian cancer: a minireview. Int J Mol Sci. 2017;18:2171. doi:10.3390/ijms18102171

28. Ricci F, Affatato R, Carrassa L, Damia G. Recent insights into mucinous ovarian carcinoma. Int J Mol Sci. 2018;19:E1569. doi:10.3390/ijms 19061569

29. Mikuła-Pietrasik J, Sosińska P, Murias M, et al. High potency of a novel resveratrol derivative, 3,3',4,4'-tetrahydroxy-trans-stilbene, against ovarian cancer is associated with an oxidative stress-mediated imbalance between DNA damage accumulation and repair. Oxid Med Cell Longev. 2015;2015:135691. doi:10.1155/2015/659750

30. Zhong LX, Li H, Wu ML, et al. Inhibition of STAT3 signaling as critical molecular event in resveratrol-suppressed ovarian cancer cells. J Ovarian Res. 2015;8:25. doi:10.1186/s13048-015-0152-4

31. Zhong LX, Zhang Y, Wu ML, et al. Resveratrol and STAT inhibitor enhance autophagy in ovarian cancer cells. Cell Death Discovery. 2016;2:15071. doi:10.1038/cddiscovery.2016.9 
32. Schlappe BA, Mueller JJ, Zivanovic O, et al. Cited rationale for variance in the use of primary intraperitoneal chemotherapy following optimal cytoreduction for stage III ovarian carcinoma at a high intraperitoneal chemotherapy utilization center. Gynecol Oncol. 2016;142:13-18. doi:10.1016/j.ygyno.2016.05.015

33. Gambini J, Inglés M, Olaso G, et al. Properties of resveratrol: in vitro and in vivo studies about metabolism, bioavailability, and biological effects in animal models and humans. Oxid Med Cell Longev. 2015;2015:837042. doi:10.1155/2015/659750

34. Sehouli J, Reinthaller A, Marth C, et al. Intra- and postoperative catumaxomab in patients with epithelial ovarian cancer: safety and two-year efficacy results from a multicentre, single-arm, phase II study. Br J Cancer. 2014;111:1519-1525. doi:10.1038/bjc.2014.348

35. Barber EL, Dusetzina SB, Stitzenberg KB, et al. Variation in neoadjuvant chemotherapy utilization for epithelial ovarian cancer at high volume hospitals in the United States and associated survival. Gynecol Oncol. 2017;145:500-507. doi:10.1016/j.ygyno.2017.03.014
36. Ornelas A, McCullough CR, Lu Z, et al. Millward. Induction of autophagy by ARHI (DIRAS3) alters fundamental metabolic pathways in ovarian cancer models. BMC Cancer. 2016;16:824. doi:10.1186/s12885-016-2850-8

37. Badgwell DB, Lu Z, Le K, et al. The tumor suppressor gene ARHI (DIRAS3) suppresses ovarian cancer cell migration through inhibition of the Stat3 and FAK/Rho signaling pathways. Oncogene. 2012;31:68-79. doi:10.1038/onc.2011.627

38. Levy C, Nechushtan H, Razin E. A new role for the STAT3 inhibitor, PIAS3: a repressor of microphthalmia transcription factor. $J$ Biol Chem. 2002;277:1962-1966. doi:10.1074/jbc. M109236200

\section{Publish your work in this journal}

Cancer Management and Research is an international, peer-reviewed open access journal focusing on cancer research and the optimal use of preventative and integrated treatment interventions to achieve improved outcomes, enhanced survival and quality of life for the cancer patient.
The manuscript management system is completely online and includes a very quick and fair peer-review system, which is all easy to use. Visit http://www.dovepress.com/testimonials.php to read real quotes from published authors. 